\title{
Testing Yukawa unification at LHC Run-3 and HL-LHC
}

\author{
Mario E. Gómez, ${ }^{a}$ Qaisar Shafi ${ }^{b}$ and Cem Salih Ün ${ }^{c}$ \\ ${ }^{a}$ Departamento de Ciencias Integradas y Centro de Estudios Avanzados \\ en Fúsica Matemáticas y Computación, Campus del Carmen, \\ Universidad de Huelva, Huelva 21071, Spain \\ ${ }^{b}$ Bartol Research Institute, Department of Physics and Astronomy, University of Delaware, \\ Newark, DE 19716, U.S.A. \\ ${ }^{c}$ Department of Physics, Bursa Uluda $\tilde{g}$ University, \\ TR16059 Bursa, Turkey \\ E-mail: mario.gomez@dfa.uhu.es, shafi@bartol.udel.edu, \\ cemsalihun@uludag.edu.tr
}

Abstract: We explore $t$ - $b$ - $\tau$ Yukawa unification $(\mathrm{YU})$ in a supersymmetric $\mathrm{SU}(4)_{c} \times$ $\mathrm{SU}(2)_{L} \times \mathrm{SU}(2)_{R}$ model without imposing a discrete left-right (L-R) symmetry. A number of interesting solutions that are compatible with $t-b-\tau$ YU, LSP neutralino dark matter (DM), and LHC and other experimental constraints are identified. In particular, they include gluino-neutralino and stau-neutralino co-annihilation scenarios, where the NLSP gluino mass can range from 1-3 TeV. Higgsino-like dark matter solutions are also identified for which gluino masses can approach $5 \mathrm{TeV}$ or so. This scenario will be tested at LHC Run-3 and its future upgrades.

KEYWORDS: Supersymmetry Phenomenology

ARXIV EPRINT: 2002.07517 


\section{Contents}

1 Introduction 1

2 Scanning procedure and experimental constraints 2

$3 t-b-\tau$ YU and DM Implications $\quad 4$

$\begin{array}{lll}4 \text { Conclusion } & 10\end{array}$

\section{Introduction}

Third family $t-b-\tau$ Yukawa Unification (YU) $[1-3]$ arises naturally in the simplest versions of supersymmetric $\mathrm{SO}(10)$ and $\mathrm{SU}(4)_{c} \times \mathrm{SU}(2)_{L} \times \mathrm{SU}(2)_{R}(4-2-2)$ models and it has attracted a fair amount of attention in recent years [4-30]. Most work on the implications of YU have assumed the presence of a discrete Left-Right (LR) symmetry (more precisely C-parity, also known as D-parity), which restricts the number of Soft Supersymmetry Breaking (SSB) parameters. It was also shown that $t-b-\tau$ YU allows the gluino NLSP solutions in 4-2-2 [3135] models with LR symmetry. In this case the LSP neutralino can provide the desired dark matter (DM) abundance, but the gluino turns out to be not much heavier than a $\mathrm{TeV}$ or so. Switching from $t-b-\tau$ YU to $b-\tau$ YU also allows stop NLSP solutions with $m_{\tilde{t}_{1}} \lesssim 1 \mathrm{TeV}[36]$.

The spontaneous breaking of $\mathrm{SO}(10)$ to its maximal subgroup 4-2-2 can be accomplished either with a Higgs 54-plet or 210-plet. The breaking with 54-plet leaves the LR symmetry unbroken [37-39]. However, using the 210-plet yields 4-2-2 symmetry but the C-parity in this case is absent [40]. The spontaneous breaking of LR symmetry also avoids a potential domain wall problem [38]. Recent works [41, 42] have discussed the sparticle spectroscopy, DM implications and muon $g-2$ in 4-2-2 with broken LR symmetry in the softly broken scalar sector without imposing the $t-b-\tau$ YU condition.

In the case of broken LR symmetry the universality between the $\mathrm{SU}(2)_{L}$ and $\mathrm{SU}(2)_{R}$ gauginos does not hold, i.e. $M_{2 L} \neq M_{2 R}$. Besides, the symmetrical structure of 4-2-2 also allows non-universality among the other gauginos as

$$
M_{1}=\frac{3}{5} M_{2 R}+\frac{2}{5} M_{3} .
$$

Despite non-universal gaugino masses, the gauge coupling unification can be maintained if 4-2-2 breaks to the MSSM gauge group at the grand unification scale $\left(M_{\mathrm{GUT}}\right)$. We should also note the fact that the presence of Higgs 210-plet, in general, breaks YU [43]. However, $t-b-\tau$ YU can be largely preserved if the third family matter fields acquire masses from the $(1,2,2)$ components of the effective MSSM Higgs doublets [44]. 
In this paper we explore the low energy consequences of imposing t-b- $\tau$ YU in a supersymmetric 4-2-2 model without assuming LR symmetry in the softly broken scalar and gaugino sectors. We employ a variety of constraints from collider physics, rare $B$-meson decays and DM searches, and we require that the LSP neutralino saturates the dark matter limits set by the Planck satellite experiment. The rest of the paper is organized as follows. We briefly describe in section 2 the scanning procedure and the experimental constraints. Section 3 discusses the low energy implications if $t-b-\tau$ YU is imposed at $M_{\mathrm{GUT}}$ and present some benchmark points to exemplify our findings. In section 4 we summarize our conclusions.

\section{Scanning procedure and experimental constraints}

We employ the ISAJET 7.84 package [45] to perform random scans over the parameter space given below. In this package, the weak scale values of gauge and third generation Yukawa couplings are evolved to $M_{\mathrm{GUT}}$ via the MSSM renormalization group equations (RGEs) in the $\overline{D R}$ regularization scheme. We apply the gauge coupling unification condition approximately as $g_{3} \approx g_{2}=g_{1}$, since $g_{3}$ can deviate from the unification by a few percent due to unknown GUT scale threshold corrections [46-48]. We only accept solutions in which $g_{3}$ deviates from unification up to $3 \%$, at most. With the boundary conditions given at $M_{\mathrm{GUT}}$, all the SSB parameters, along with the gauge and Yukawa couplings, are evolved back to the weak scale $M_{\mathrm{Z}}$.

In evaluating Yukawa couplings the SUSY threshold corrections [49] are taken into account at the common scale $M_{\mathrm{SUSY}}=\sqrt{m_{\tilde{t}_{L}} m_{\tilde{t}_{R}}}$. The entire parameter set is iteratively run between $M_{\mathrm{Z}}$ and $M_{\mathrm{GUT}}$ using the full 2-loop RGEs until a stable solution is obtained. To better account for leading-log corrections, one-loop step-beta functions are adopted for gauge and Yukawa couplings, and the SSB parameters $m_{i}$ are extracted from RGEs at appropriate scales $m_{i}=m_{i}\left(m_{i}\right)$. The RGE-improved 1-loop effective potential is minimized at an optimized scale $M_{\mathrm{SUSY}}$, which effectively accounts for the leading 2-loop corrections. Full 1-loop radiative corrections are incorporated for all sparticle masses.

We have scanned the parameter space of 4-2-2 with broken LR symmetry in both the scalar and gaugino sectors. The fundamental parameters in this framework and their ranges are as follows:

$$
\begin{aligned}
& 0.1 \leq m_{L} \quad \leq 10 \mathrm{TeV} \\
& 0.05 \leq M_{2 L} \quad \leq 5 \mathrm{TeV} \\
& -3 \leq M_{3} \quad \leq 5 \mathrm{TeV} \\
& -3 \leq A_{0} / m_{L} \leq 3 \\
& 2 \leq \tan \beta \leq 65 \\
& -3 \leq \mathrm{x}_{\mathrm{LR}} \quad \leq 3 \\
& -3 \leq \mathrm{y}_{\mathrm{LR}} \quad \leq 3 \\
& 0 \leq \mathrm{x}_{\mathrm{d}} \quad \leq 3 \\
& -1 \leq \mathrm{x}_{\mathrm{u}} \quad \leq 2 \text {. }
\end{aligned}
$$


Here $m_{L}$ is the universal SSB mass term for the left-handed SUSY scalars, while $M_{2}$ and $M_{3}$ are the SSB gaugino mass terms. $A_{0}$ denotes the SSB trilinear scalar interaction term, and $\tan \beta$ is the ratio of the vacuum expectation values of the MSSM Higgs doublets such that $\tan \beta \equiv v_{u} / v_{d} . \quad \mathrm{x}_{\mathrm{LR}}$ measures the LR breaking in the scalar sector with $m_{2 R}=$ $\mathrm{x}_{\mathrm{LR}} m_{L}$, where $m_{2 R}$ is the SSB mass term for the right-handed SUSY scalars. Similarly $\mathrm{y}_{L R}$ parametrizes the LR breaking in the gaugino sector as $M_{2 R}=\mathrm{y}_{\mathrm{LR}} M_{2 L}$. We also employ non-universal SSB masses for the MSSM Higgs fields by setting $m_{H_{d}}=x_{d} m_{L}$ and $m_{H_{u}}=x_{u} m_{L}$.

In scanning the parameter space, we employ the Metropolis-Hastings algorithm as described in $[50,51]$. The data points collected all satisfy the requirement of REWSB, with the neutralino in each case being the LSP. After collecting data, we impose the mass bounds on all particles [52] including the current bounds on the Higgs boson [53, 54] and the gluino [55]. In addition, we use the IsaTools package [56-61] to implement the phenomenological constraints from rare $B$-meson decays such as those from $\operatorname{BR}\left(B_{s} \rightarrow\right.$ $\left.\mu^{+} \mu^{-}\right)$[62], $\mathrm{BR}\left(B_{s} \rightarrow X_{s} \gamma\right)$ [63] and $\mathrm{BR}\left(B_{u} \rightarrow \tau \nu\right)$ [64]. We also apply the Planck bound on the relic density of LSP neutralino within $5 \sigma$ [65]. The following experimental constraints along with their uncertainties are employed in our analyses:

$$
\begin{aligned}
m_{h} & =123-127 \mathrm{GeV} \\
m_{\tilde{g}} & \geq 2.1 \mathrm{TeV}(\geq 0.8 \mathrm{TeV} \text { if gluino is NLSP }) \\
0.8 \times 10^{-9} & \leq \mathrm{BR}\left(B_{s} \rightarrow \mu^{+} \mu^{-}\right) \leq 6.2 \times 10^{-9}(2 \sigma) \\
2.99 \times 10^{-4} \leq \mathrm{BR}\left(B \rightarrow X_{s} \gamma\right) \leq 3.87 \times 10^{-4}(2 \sigma) & \\
0.15 & \leq \frac{\mathrm{BR}\left(B_{u} \rightarrow \tau \nu_{\tau}\right)_{\mathrm{MSSM}}}{\mathrm{BR}\left(B_{u} \rightarrow \tau \nu_{\tau}\right)_{\mathrm{SM}}} \leq 2.41(3 \sigma) \\
0.114 & \leq \Omega_{\mathrm{CDM}} h^{2} \leq 0.126(5 \sigma)
\end{aligned}
$$

In addition to these constraints, we quantify $t-b-\tau$ YU with the parameter $R_{t b \tau}$ as

$$
R_{t b \tau} \equiv \frac{\operatorname{Max}\left(y_{t}, y_{b}, y_{\tau}\right)}{\operatorname{Min}\left(y_{t}, y_{b}, y_{\tau}\right)}
$$

where $R_{t b \tau}=1$ means perfect $t-b-\tau$ YU. However, considering various uncertainties we consider solutions to be compatible with $t-b-\tau$ YU for $R_{t b \tau} \leq 1.1$.

After the mass spectrum and DM implications are calculated, it is interesting to confront the predictions from the models consistent with the constraints given in eq. (2.2) as well as $t-b-\tau$ YU with the LHC bounds and future prospects. To this end, we employ the tools provided by the Smodels-v1.2.2. [66-69]. This package decomposes the theoretical models into the Simplified Model Spectra (SMS) which are compared with the data provided by the ATLAS and CMS collaborations [70, 71]. For each model, we use SUSYHIT [72] to compute the decay ratios of the SUSY particles and PYTHIA [73] to produce the corresponding cross sections. 

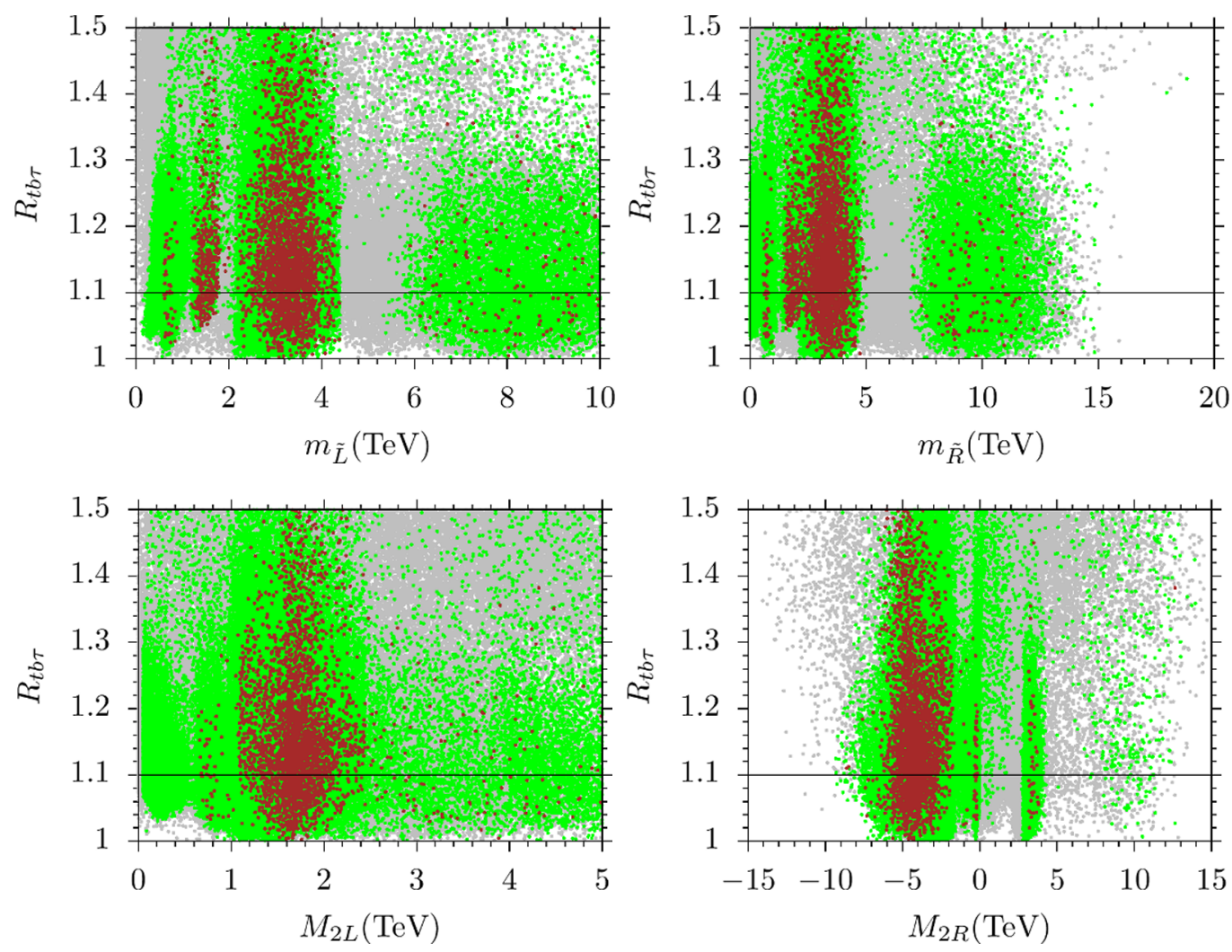

Figure 1. Plots in the $R_{t b \tau}-m_{\tilde{L}}, R_{t b \tau}-m_{\tilde{R}}, R_{t b \tau}-M_{2 L}$ and $R_{t b \tau}-M_{2 R}$ planes. All points are comparible with the REWSB and LSP neutralino conditions. Green points satisfy the mass bounds and constraints from rare $B$-meson decays. Brown points form a subset of green and they yield relic abundance of LSP neutralino consistent with the Planck measurements within $5 \sigma$. The regions below the horizontal lines correspond to $R_{t b \tau}=1.1$ are considered as to be compatible with the $t-b-\tau$ Yukawa unification.

\section{$3 \quad t-b-\tau$ YU and DM Implications}

In this section we present our results for $t-b-\tau$ YU within the 4-2-2 framework. Figure 1 displays the fundamental parameter space of $t-b-\tau$ YU in terms of the SSB scalar (top panels) and gaugino (bottom panels) mass terms with plots in the $R_{t b \tau}-m_{\tilde{L}}, R_{t b \tau}-m_{\tilde{R}}$, $R_{t b \tau}-M_{2 L}$ and $R_{t b \tau}-M_{2 R}$ planes. All points are compatible with REWSB and LSP neutralino conditions. Green points satisfy the mass bounds and constraints from rare $B$-meson decays. Brown points form a subset of green and they yield relic abundance of LSP neutralino consistent with the Planck measurements within $5 \sigma$. The horizontal line indicates the region with $R_{t b \tau}=1.1$, and points below this line are considered as being in very good agreement with the $t-b-\tau$ Yukawa unification. The $R_{t b \tau}-m_{\tilde{L}}$ plane shows that $t-b-\tau$ YU can be realized within a wide range of $m_{\tilde{L}}$ from about $400 \mathrm{GeV}$ to $10 \mathrm{TeV}$. Two regions can be identified, which are separated by a gray area. The gray region between them is excluded mostly by the gluino mass bound, which will be shown in more detail later. The SSB mass term for the right-handed scalar particles can be as light as about $1 \mathrm{TeV}$, while $t-b-\tau \mathrm{YU}$ condition restricts it at about $15 \mathrm{TeV}$ from above, as seen from the 

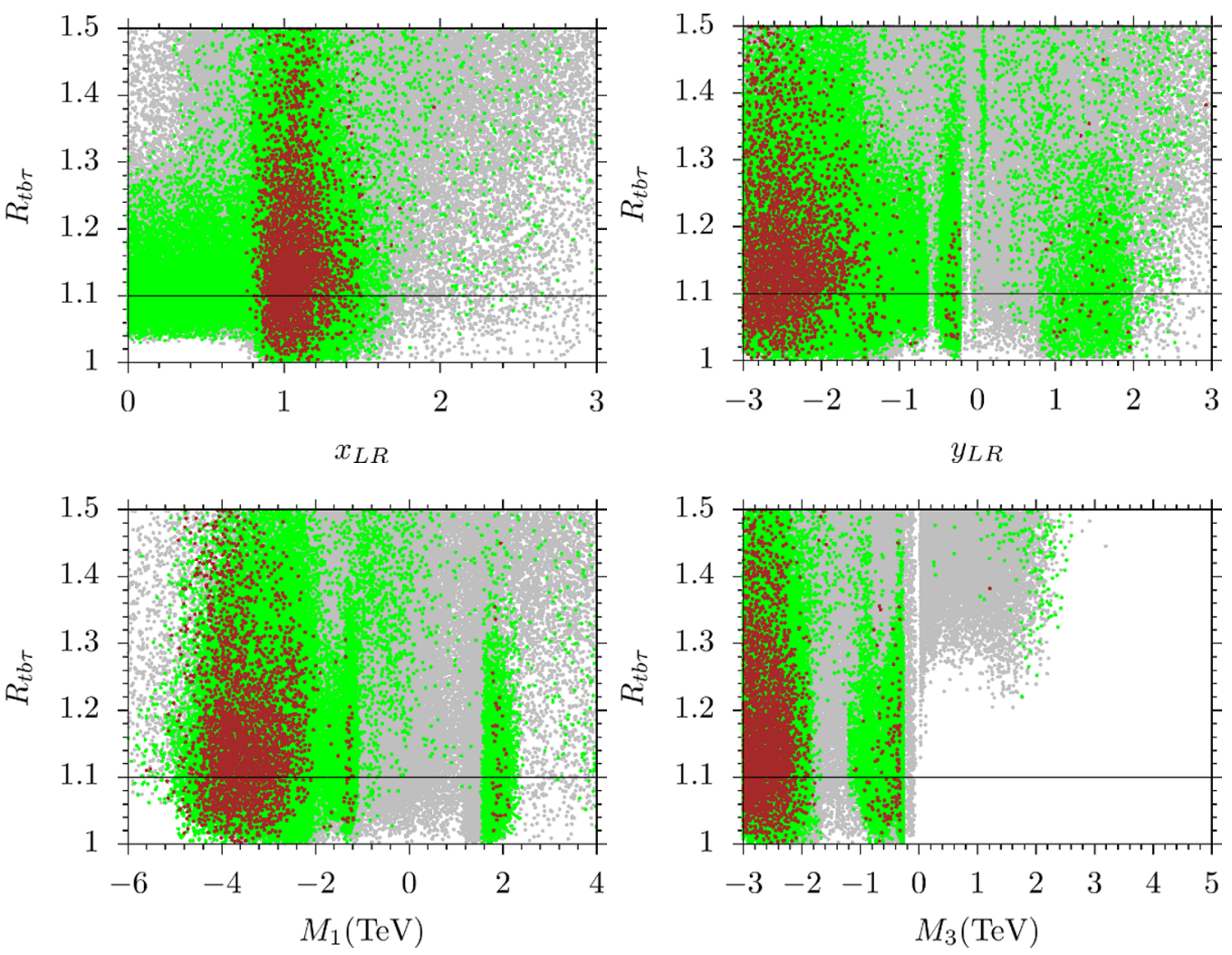

Figure 2. Plots in the $R_{t b \tau}-x_{L R}, R_{t b \tau}-y_{L R}, R_{t b \tau}-M_{1}$ and $R_{t b \tau}-M_{3}$ planes. The color coding is the same as in figure 1.

$R_{t b \tau}-m_{\tilde{R}}$ plane. The left-bottom plane displays the SSB mass term for $M_{2 L}$, and as is seen, the $t-b-\tau$ YU solutions can be realized in a wide range of values for $M_{2 L}$ in our scan. It is bounded at about $800 \mathrm{GeV}$ from below by the DM constraints. The mass term for the $\mathrm{SU}(2)_{R}$ gaugino could lie in a wider range from about $-10 \mathrm{TeV}$ to $4 \mathrm{TeV}$ consistent with all the LHC and DM constraints.

The parameters quantifying non-universality in the scalar and gaugino sectors are shown in figure 2 along with the SSB gaugino mass terms with plots in the $R_{t b \tau}-x_{L R}$, $R_{t b \tau}-y_{L R}, R_{t b \tau}-M_{1}$ and $R_{t b \tau}-M_{3}$ planes. The color coding is the same as in figure 1 . Despite its wider range in our scan, the $R_{t b \tau}-x_{L R}$ plane shows that LR breaking in the scalar sector can be realized consistent with the DM constraints if $0.8 \lesssim x_{L R} \lesssim 1.6$. Note that $x_{L R}=1$ restores the symmetry in the scalar sector. On the other hand, the LR breaking in the gaugino sector can be crucial for $t-b-\tau$ YU, as seen from the $R_{t b \tau}-y_{L R}$ plane with $\left|y_{L R}\right|$ as large as 3. Even though $t-b-\tau$ YU mostly prefers $y_{L R}$ to be negative, it is possible to realize positive $y_{L R}$ values in a relatively small portion of the parameter space, which also leads to negative $M_{2 R}$ in most of the parameter space. Its impact can be seen from the $R_{t b \tau}-M_{1}$ plane where $M_{1}$ is mostly negative and its magnitude can be as high as about $5 \mathrm{TeV}$, while it is restricted to $M_{1} \sim 2 \mathrm{TeV}$ in the positive region. Since $M_{1}$ controls the bino mass at the low scale, such large values of $M_{1}$ prevent bino from being the LSP. The parameter $M_{3}$ is shown in the right-bottom panel, and it is seen that $t-b-\tau$ 

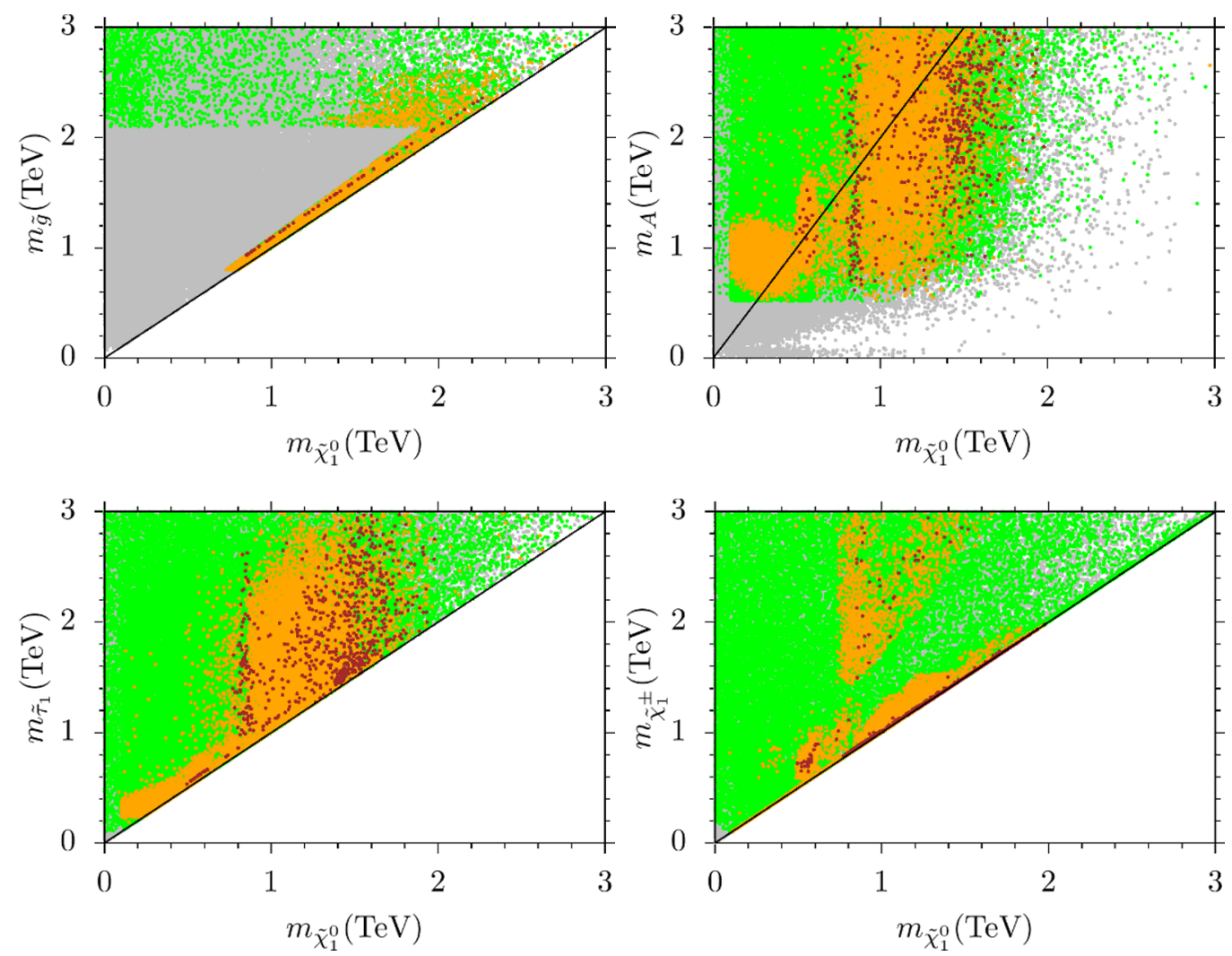

Figure 3. Plots in the $m_{\tilde{g}}-m_{\tilde{\chi}_{1}^{0}}, m_{A}-m_{\tilde{\chi}_{1}^{0}}, m_{\tilde{\tau}_{1}}-m_{\tilde{\chi}_{1}^{0}}$ and $m_{\tilde{\chi}_{1}^{ \pm}}-m_{\tilde{\chi}_{1}^{0}}$ planes. All points are compatible with the REWSB and LSP neutralino conditions. Green points satisfy the mass bounds and constraints from rare $B$-meson decays. Orange points form a subset of green and they are compatible with $t-b-\tau$ Yukawa unification. Brown points are a subset of orange, they are consistent with the Planck bound on the relic abundance of LSP neutralino within $5 \sigma$. The diagonal lines indicate regions in which the displayed particles are degenerate in mass, except for the line in the $m_{A}-m_{\tilde{\chi}_{1}^{0}}$ plane which shows the solutions with $m_{A}=2 m_{\tilde{\chi}_{1}^{0}}$.

YU condition allows only negative $M_{3}$ values. In this context, according to eq. (1.1), one can expect very large $M_{2 L}$ for $M_{1}>0$; thus this region is most likely to realize a bino or Higgsino DM.

We present the low scale mass spectrum in figure 3 with plots in the $m_{\tilde{g}}-m_{\tilde{\chi}_{1}^{0}}$, $m_{A}-m_{\tilde{\chi}_{1}^{0}}, m_{\tilde{\tau}_{1}}-m_{\tilde{\chi}_{1}^{0}}$ and $m_{\tilde{\chi}_{1}^{ \pm}}-m_{\tilde{\chi}_{1}^{0}}$ planes. All points are compatible with REWSB and LSP neutralino conditions. Green points satisfy the mass bounds and constraints from rare $B$-meson decays. Orange points form a subset of green and they are compatible with $t-b-\tau$ YU. Brown points are a subset of orange and they are consistent with the Planck bound on the relic abundance of LSP neutralino within $5 \sigma$. The diagonal lines indicate regions in which the displayed particles are degenerate in mass, and the line in the $m_{A}-m_{\tilde{\chi}_{1}^{0}}$ plane depicts solutions with $m_{A}=2 m_{\tilde{\chi}_{1}^{0}}$. Some of the most interesting results correspond to NLSP gluino solutions. In previous studies NLSP gluino masses of order $1 \mathrm{TeV}$ are found, compatible with $t-b-\tau$ YU in the presence of LR symmetry. However, the $m_{\tilde{g}}-m_{\tilde{\chi}_{1}^{0}}$ plane shows that in our case one can realize NLSP gluino solutions compatible with $t-b-\tau$ YU 

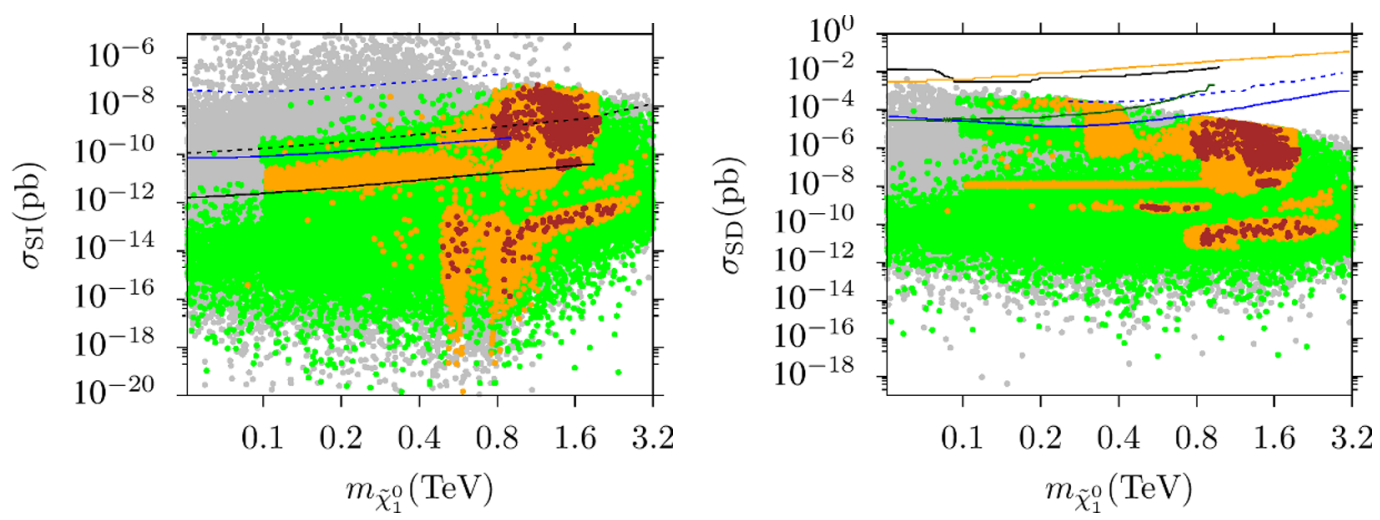

Figure 4. Spin-independent (left) and spin-dependent (right) scattering cross-sections versus the LSP neutralino mass. In the $\sigma_{S I}-m_{\tilde{\chi}_{1}^{0}}$ plane, the dashed (solid) blue line represents the current (future) results from the SuperCDMS experiment [74]. The dashed (solid) black line indicates the current (future) results from the LUX-Zeplin experiment [75]. In the $\sigma_{S D}-m_{\tilde{\chi}_{1}^{0}}$ plane, the solid black line represents the currrent bound from Super-K [76], and the solid orange line is set by the LUX results [77]. The green line is obtained from collider analyses [78], and the dashed (solid) blue line shows the current (future) results from IceCube DeepCore.

for gluino mass scales up to about 2.5 TeV. Moreover, as seen from the other panels of figure 3 , the mass spectra also favor the $A$-resonance solution if $1 \lesssim m_{A} \lesssim 3 \mathrm{TeV}$, and stau-neutralino and chargino-neutralino coannihilation solutions if $0.6 \lesssim m_{\tilde{\tau}_{1}} \lesssim 1.6 \mathrm{TeV}$. The $m_{\tilde{\chi}_{1}^{ \pm}}-m_{\tilde{\chi}_{1}^{0}}$ plane also shows that the lightest chargino is almost as light as the LSP neutralino in most of the parameter space. Approximate mass degeneracy between the lightest chargino and LSP neutralino is one of the characteristics features of DM composed of wino or Higgsino.

With a wino and/or Higgsino DM, one can expect large cross-section in the DM scattering processes. For wino DM, the scattering off nuclei occurs through $\mathrm{SU}(2)$ interactions, while Yukawa interactions take part if the Higgsino happens to be the LSP. Figure 4 shows results for the spin-independent (left) and spin-dependent (right) scattering cross-sections versus the LSP neutralino mass. In the $\sigma_{S I}-m_{\tilde{\chi}_{1}^{0}}$ plane, the dashed (solid) blue line represents the current (future) results from the SuperCDMS experiment [74]. The dashed (solid) black line indicates the current (future) results from the LUX-Zeplin experiment [75]. In the $\sigma_{S D}-m_{\tilde{\chi}_{1}^{0}}$ plane, the solid black line represents the currrent bound from Super-K [76], and the solid orange line is set by the LUX results [77]. The green line is obtained from collider analyses [78], and the dashed (solid) blue line shows the current (future) results from IceCube/DeepCore. The region in the $\sigma_{\mathrm{SI}}-m_{\tilde{\chi}_{1}^{0}}$ plane, which is cut by the dashed black exclusion curve of the current results from the LUX measurements, implies a Higgsino DM. Even though many solutions are already excluded due to large scattering cross-sections, it is still possible to realize a Higgsino DM slightly below this curve. These solutions are expected to be probed in the direct detection DM experiments in the near future. The solutions between the current and future exclusion curves also yield a wino DM, and they are within reach of future experiments. The remaining solutions lying below all the curves represent the DM in which bino is involved. 
Most of the models allowed by the constraints listed in eq. (2.2) and compatible with $t-b-\tau$ YU present topologies that cannot be detected at the LHC according to the Smodels analysis. Note that this is the case for the subset of models satisfying the DM constraint. Although many models predict relatively low SUSY masses, their signals escape the LHC bounds as can be understood from our previous discussions. For instance, although the constraints on gluino masses are quite severe, we can find cases with gluino masses of order $800 \mathrm{GeV}$ compatible with $t-b-\tau$ YU. However, the DM constraint bound it at about $900 \mathrm{GeV}$ from below, as seen in figure 3. In this region, the gluino happens to be the NLSP, and it can decay into a LSP neutralino along with a quark-antiquark pair from the first two families. The bound from these processes is set as $m_{\tilde{g}} \gtrsim 800 \mathrm{GeV}[79,80]$. Figure 3 also shows that the stau can be as light as $500 \mathrm{GeV}$ or so, consistent with all the constraints and $t-b-\tau$ YU, where the LSP neutralino is formed mostly from bino and/or wino. If the charginos and second lightest neutralino are allowed to decay into staus, YU with $m_{\tilde{\tau}} \lesssim 350 \mathrm{GeV}$ and/or $m_{\tilde{\chi}_{1}^{ \pm}} \lesssim 1.1 \mathrm{TeV}$ is excluded [81-83]. On the other hand, the lightest stau in MSSM is mostly composed of the right-handed stau, which forbids the lightest chargino to decay into a stau. Besides, as seen from the chargino and LSP neutralino masses shown in the right bottom panel of figure 3 , the chargino decay into a LSP neutralino along with a $W$-boson is not kinematically allowed, which also loosens the constraints on the chargino and stau masses.

Before concluding the discussion about $t-b-\tau$ YU, we present five benchmark points that are compatible with the experimental constraints. All masses are given in $\mathrm{GeV}$, and the masses shown in red indicate the mass degeneracy with the LSP neutralino within $10 \%$, except Point 3, in which the red color emphasizes the mass relation between the neutral Higgs bosons and LSP neutralino, namely $m_{H, A} \simeq 2 m_{\tilde{\chi}_{1}^{0}}$. Points 1 and 2 depict solutions for the gluino-neutralino coannihilation scenario with NLSP gluino masses of about 933 and $2357 \mathrm{GeV}$, respectively. Point 3 represents an $A$-resonance solution. The DM relic abundance is saturated with bino-like LSP neutralino in the first three points. Point 4 displays a wino-like DM solution where the lightest chargino mass is close to the wino and also to the lightest stau mass. Coannihilations of these three species lead to the desired relic DM abundance. Point 5 is a typical solution for Higgsino-like DM with the chargino and neutralino masses given as $m_{\tilde{\chi}_{1}^{ \pm}} \sim m_{\tilde{\chi}_{2}^{0}} \sim m_{\tilde{\chi}_{1}^{0}}$. The spin-independent cross-section is calculated to be slightly below the current LUX result and so this scenario will be tested relatively soon.

In addition, Point 3 in table 1 depicts solutions with relatively light Higgs bosons whose masses are about $1 \mathrm{TeV}$. Such solutions are severely constrained if these Higgs bosons mostly decay into leptons $[84,85]$. On the other hand, the solutions exemplified with Point 3 predict that these Higgs bosons mostly decays into top and bottom quarks with $\mathrm{BR}(H, A) \rightarrow b \bar{b} \approx \mathrm{BR}\left(H^{ \pm} \rightarrow t b\right) \simeq 85 \%$. The analyses for these decay channels lead to the mass bounds as $m_{H, A, H^{ \pm}} \gtrsim 800 \mathrm{GeV}$ for moderate $\tan \beta$ values [86, 87]. In this context, the predictions of our model can also be tested through the decay channels of the extra Higgs bosons in Run-3 and HL-LHC. 


\begin{tabular}{|c|c|c|c|c|c|}
\hline & Point 1 & Point 2 & Point 3 & Point 4 & Point 5 \\
\hline$m_{\tilde{L}}$ & 8031 & 9461 & 781 & 1202 & 3714 \\
\hline$M_{1}$ & 1786 & -4810 & -1288 & -3653 & -4502 \\
\hline$M_{2 L}$ & 2859 & 3915 & 758.5 & 1559 & 2348 \\
\hline$M_{3}$ & -316.7 & -926 & -2904 & -2802 & -2537 \\
\hline$A_{0} / m_{\tilde{L}}$ & -1.06 & -0.13 & 1.57 & 1.16 & 1.30 \\
\hline $\tan \beta$ & 48.0 & 47.8 & 43.8 & 42.6 & 52.4 \\
\hline$x_{L R}$ & 1.43 & 0.78 & 0.83 & 1.45 & 0.84 \\
\hline$y_{L R}$ & 1.11 & -1.89 & -0.28 & -2.71 & -2.47 \\
\hline$m_{\tilde{R}}$ & 11460 & 7398 & 646.9 & 1740 & 3111 \\
\hline$M_{2 R}$ & 3188 & -7399 & -210.1 & -4221 & -5811 \\
\hline$\mu$ & 8416 & 6401 & 3431 & 3398 & 894.4 \\
\hline$m_{h}$ & 124.3 & 123.2 & 123.2 & 123.1 & 124.1 \\
\hline$m_{H}$ & 6205 & 5568 & 1088 & 1549 & 2432 \\
\hline$m_{A}$ & 6164 & 5531 & 1081 & 1539 & 2417 \\
\hline$m_{H^{ \pm}}$ & 6206 & 5568 & 1093 & 1552 & 2434 \\
\hline$m_{\tilde{\chi}_{1}^{0}}, m_{\tilde{\chi}_{2}^{0}}$ & $849.6,2530$ & 2259,3438 & $558.1,711.6$ & 1387,1638 & $850.6,853.3$ \\
\hline$m_{\tilde{\chi}_{3}^{0}}, m_{\tilde{\chi}_{4}^{0}}$ & 7757,7757 & 5962,5962 & 3190,3190 & 3165,3165 & 2044,2058 \\
\hline$m_{\tilde{\chi}_{1}^{ \pm}}, m_{\tilde{\chi}_{2}^{ \pm}}$ & 2535,7710 & 3439,5962 & $713.8,3162$ & 1389,3137 & $871.5,2018$ \\
\hline$M_{\tilde{g}}$ & 933.9 & 2357 & 5954 & 5781 & 5360 \\
\hline$m_{\tilde{u}_{L}}, m_{\tilde{u}_{R}}$ & 8183,11379 & 9906,7462 & 5172,5145 & 5163,5294 & 6019,5540 \\
\hline$m_{\tilde{t}_{1}}, m_{\tilde{t}_{2}}$ & 4075,9113 & 4763,7707 & 4371,4506 & 4331,4564 & 3523,4338 \\
\hline$m_{\tilde{d}_{L}}, m_{\tilde{d}_{R}}$ & 8183,11558 & 9906,7623 & 5173,5151 & 5164,5258 & 6019,5516 \\
\hline$m_{\tilde{b}_{1}}, m_{\tilde{b}_{2}}$ & 4131,8784 & 3491,7690 & 4340,4490 & 4327,4466 & 3556,4327 \\
\hline$m_{\tilde{\nu}_{e, \mu}}, m_{\tilde{\nu}_{\tau}}$ & 8104,6498 & 9720,8794 & $909.3,905.6$ & 1668,1449 & 4042,3245 \\
\hline$m_{\tilde{e}_{L}}, m_{\tilde{e}_{R}}$ & 8110,11641 & 9714,7811 & $914.7,843.7$ & 1667,2236 & 4043,3600 \\
\hline$m_{\tilde{\tau}_{1}}, m_{\tilde{\tau}_{2}}$ & 6509,9396 & 5167,8783 & $605.6,988.1$ & 1436,1896 & 1178,3245 \\
\hline$\sigma_{\mathrm{SI}}(p b)$ & $0.40 \times 10^{-14}$ & $0.71 \times 10^{-12}$ & $0.13 \times 10^{-13}$ & $0.47 \times 10^{-10}$ & $0.19 \times 10^{-9}$ \\
\hline$\sigma_{\mathrm{SD}}(p b)$ & $0.16 \times 10^{-10}$ & $0.48 \times 10^{-10}$ & $0.68 \times 10^{-9}$ & $0.17 \times 10^{-7}$ & $0.29 \times 10^{-6}$ \\
\hline$\Omega h^{2}$ & 0.116 & 0.124 & 0.122 & 0.120 & 0.125 \\
\hline$R_{t b \tau}$ & 1.04 & 1.08 & 1.09 & 1.08 & 1.09 \\
\hline
\end{tabular}

Table 1. Benchmark points are compatible with all experimental constraints used in this paper. All points are chosen to be allowed by the constraints. All masses are given in GeV. Points 1 and 2 depict NLSP gluino solutions and point 3 represents an $A$-resonance solution. The first three points predict bino-like DM. Point 4 displays a stau-neutralino coannihilation solution with a wino-like DM. Point 5 is a solution with a Higgsino-like DM and with $m_{\tilde{\chi}_{1}^{ \pm}} \sim m_{\tilde{\chi}_{2}^{0}} \sim m_{\tilde{\chi}_{1}^{0}}$. 


\section{Conclusion}

We have explored the LHC and DM implications of $t-b-\tau$ YU in the supersymmetric 4-2-2 framework without imposing a discrete LR symmetry. We only accept solutions which yield one of the neutralinos as the LSP that saturates the DM abundance. We identify the gluinoneutralino coannihilation scenarios, and present consistent solutions for $m_{\tilde{g}} \lesssim 2.4 \mathrm{TeV}$. Without the NLSP constraint the gluino can be as heavy as about $6 \mathrm{TeV}$, which can be probed at the LHC and future colliders. In addition to the gluino-neutralino coannihilation scenario, some $A$-resonance solutions are also identified with $1 \lesssim m_{A} \lesssim 3 \mathrm{TeV}$. Similarly, the stau-neutralino and chargino-neutralino coannihilation processes can be realized with the stau and chargino masses in the range $0.6 \lesssim m_{\tilde{\tau}}, m_{\tilde{\chi}_{1}^{ \pm}} \lesssim 2 \mathrm{TeV}$.

The 4-2-2 model also yields wino and Higgsino-like DM as well as solutions with bino DM. We observe that while many of the Higgsino DM solutions are excluded by the direct detection experiments, it is still possible to realize some solutions lying slightly below the current exclusion curves. In other words, Higgsino DM in the 4-2-2 framework will be seriously tested in the near future. Wino-like DM solutions are allowed by the current measurements, and they lie within the reach of the near future experiments.

We exemplify our findings with five benchmark points including the full spectrum for the SUSY particles. In addition to the DM implications, the stop cannot be lighter than about $3 \mathrm{TeV}$, while squarks of the first two families are relatively heavy ( $\gtrsim 5 \mathrm{TeV}$ ). In addition, we identify some solutions which predict the heavy Higgs bosons masses at about $1 \mathrm{TeV}$. These Higgs bosons mostly decay into the top and bottom quarks, and they are expected to be tested in Run-3 and HL-LHC through their hadronic decays.

\section{Acknowledgments}

We would like to thank Zafer Altın for discussions. CSU also thank Universidad de Huelva and Bartol Research Institute of University of Delaware for their kind hospitality, where part of this work has been done. The research of M.E.G. was supported by the Spanish MINECO, under grant FPA2017-86380-P. R. Q.S. acknowledges support by the DOE grant No. DE-SC0013880. The work of CSU is supported in part by the Scientific and Technological Research Council of Turkey (TUBITAK) Grant no. MFAG-118F090. Part of the calculations reported in this paper were performed at the National Academic Network and Information Center (ULAKBIM) of TUBITAK, High Performance and Grid Computing Center (TRUBA Resources). CSU also acknowledges support from the CEAFMC of the University of Huelva.

Open Access. This article is distributed under the terms of the Creative Commons Attribution License (CC-BY 4.0), which permits any use, distribution and reproduction in any medium, provided the original author(s) and source are credited.

\section{References}

[1] B. Ananthanarayan, G. Lazarides and Q. Shafi, Top mass prediction from supersymmetric guts, Phys. Rev. D 44 (1991) 1613 [InSPIRE]. 
[2] B. Ananthanarayan, G. Lazarides and Q. Shafi, Radiative electroweak breaking and sparticle spectroscopy with tan Beta approximately $=m(t) / m(b)$, Phys. Lett. B 300 (1993) 245 [INSPIRE].

[3] Q. Shafi and B. Ananthanarayan, Will LEP-2 narrowly miss the Weinberg-Salam Higgs boson?, in the proceedings of the Summer School in High-energy Physics and Cosmology, June 17-August 9, Trieste, Italy (1991).

[4] V.D. Barger, M.S. Berger and P. Ohmann, The supersymmetric particle spectrum, Phys. Rev. D 49 (1994) 4908 [hep-ph/9311269] [INSPIRE].

[5] M. Carena, M. Olechowski, S. Pokorski and C.E.M. Wagner, Electroweak symmetry breaking and bottom-top Yukawa unification, Nucl. Phys. B 426 (1994) 269 [hep-ph/9402253] [INSPIRE].

[6] B. Ananthanarayan, Q. Shafi and X.M. Wang, Improved predictions for top quark, lightest supersymmetric particle and Higgs scalar masses, Phys. Rev. D 50 (1994) 5980 [hep-ph/9311225] [INSPIRE].

[7] G. Anderson et al., Precise predictions for $m_{t}, V_{c b}$, and $\tan \beta$, Phys. Rev. D 47 (1993) R3702.

[8] G. Anderson, S. Raby, S. Dimopoulos, L.J. Hall and G.D. Starkman, A Systematic SO(10) operator analysis for fermion masses, Phys. Rev. D 49 (1994) 3660 [hep-ph/9308333] [INSPIRE].

[9] R. Rattazzi and U. Sarid, The unified minimal supersymmetric model with large Yukawa couplings, Phys. Rev. D 53 (1996) 1553 [hep-ph/9505428] [INSPIRE].

[10] T. Blazek, M. Carena, S. Raby and C.E.M. Wagner, A global $\chi^{2}$ analysis of electroweak data in SO(10) SUSY GUTs, Phys. Rev. D 56 (1997) 6919 [hep-ph/9611217] [INSPIRE].

[11] T. Blazek, S. Raby and K. Tobe, Neutrino oscillations in an $\mathrm{SO}(10)$ SUSY GUT with $\mathrm{U}(2) \times \mathrm{U}(1)^{* *} n$ family symmetry, Phys. Rev. D 62 (2000) 055001 [hep-ph/9912482] [INSPIRE].

[12] H. Baer, M.A. Diaz, J. Ferrandis and X. Tata, Sparticle mass spectra from $\mathrm{SO}(10)$ grand unified models with Yukawa coupling unification, Phys. Rev. D 61 (2000) 111701 [hep-ph/9907211] [INSPIRE].

[13] H. Baer et al., Yukawa unified supersymmetric $\mathrm{SO}(10)$ model: cosmology, rare decays and collider searches, Phys. Rev. D 63 (2000) 015007 [hep-ph/0005027] [InSPIRE].

[14] S. Profumo, Neutralino dark matter, $b-\tau$ Yukawa unification and nonuniversal sfermion masses, Phys. Rev. D 68 (2003) 015006 [hep-ph/0304071] [INSPIRE].

[15] C. Balázs and R. Dermisek, Yukawa coupling unification and nonuniversal gaugino mediation of supersymmetry breaking, JHEP 06 (2003) 024 [hep-ph/0303161] [INSPIRE].

[16] C. Pallis, $b \tau$ unification with gaugino and sfermion mass nonuniversality, Nucl. Phys. B 678 (2004) 398 [hep-ph/0304047] [INSPIRE].

[17] M.E. Gomez, G. Lazarides and C. Pallis, Supersymmetric cold dark matter with Yukawa unification, Phys. Rev. D 61 (2000) 123512 [hep-ph/9907261] [INSPIRE].

[18] M.E. Gomez, G. Lazarides and C. Pallis, Yukawa quasi-unification, Nucl. Phys. B 638 (2002) 165 [hep-ph/0203131] [INSPIRE].

[19] M.E. Gomez, G. Lazarides and C. Pallis, Yukawa quasi-unification with $\mu<0$, Phys. Rev. D 67 (2003) 097701. 
[20] I. Gogoladze, Y. Mimura, S. Nandi and K. Tobe, Test of gauge Yukawa unification, Phys. Lett. B $5 \mathbf{7 5}$ (2003) 66 [hep-ph/0307397] [INSPIRE].

[21] U. Chattopadhyay, A. Corsetti and P. Nath, Supersymmetric dark matter and Yukawa unification, Phys. Rev. D 66 (2002) 035003 [hep-ph/0201001] [INSPIRE].

[22] T. Blazek, R. Dermisek and S. Raby, Predictions for Higgs and supersymmetry spectra from SO(10) Yukawa unification with mu greater than 0, Phys. Rev. Lett. 88 (2002) 111804 [hep-ph/0107097] [INSPIRE].

[23] T. Blazek, R. Dermisek and S. Raby, Yukawa unification in SO(10), Phys. Rev. D 65 (2002) 115004 [hep-ph/0201081] [INSPIRE].

[24] M.E. Gomez, T. Ibrahim, P. Nath and S. Skadhauge, WMAP dark matter constraints and Yukawa unification in SUGRA models with CP phases, Phys. Rev. D 72 (2005) 095008 [hep-ph/0506243] [INSPIRE].

[25] K. Tobe and J.D. Wells, Revisiting top bottom $\tau$ Yukawa unification in supersymmetric grand unified theories, Nucl. Phys. B 663 (2003) 123 [hep-ph/0301015] [INSPIRE].

[26] W. Altmannshofer, D. Guadagnoli, S. Raby and D.M. Straub, SUSY GUTs with Yukawa unification: a go/no-go study using FCNC processes, Phys. Lett. B 668 (2008) 385.

[27] D. Guadagnoli, S. Raby and D.M. Straub, Viable and testable SUSY GUTs with Yukawa unification: The Case of split trilinears, JHEP 10 (2009) 059 [arXiv:0907.4709] [INSPIRE].

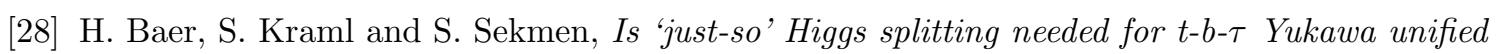
SUSY GUTs?, JHEP 09 (2009) 005 [arXiv:0908.0134] [INSPIRE].

[29] K. Choi, D. Guadagnoli, S.H. Im and C.B. Park, Sparticle masses from transverse-mass kinks at the LHC: the case of Yukawa-unified SUSY GUTs, JHEP 10 (2010) 025 [arXiv: 1005.0618] [INSPIRE].

[30] B. Dutta and Y. Mimura, Yukawa unification with four Higgs doublets in supersymmetric GUT, Phys. Lett. B 790 (2019) 589 [arXiv:1810.08413] [InSPIRE].

[31] I. Gogoladze, R. Khalid and Q. Shafi, Yukawa unification and neutralino dark matter in $\mathrm{SU}(4)(c) \times \mathrm{SU}(2)(L) \times \mathrm{SU}(2)(R)$, Phys. Rev. D 79 (2009) 115004 [arXiv:0903.5204] [INSPIRE].

[32] I. Gogoladze, R. Khalid and Q. Shafi, Coannihilation scenarios and particle spectroscopy in $\mathrm{SU}(4)(c) \times \mathrm{SU}(2)(L) \times \mathrm{SU}(2)(R)$, Phys. Rev. D 80 (2009) 095016 [arXiv:0908.0731] [INSPIRE].

[33] S. Profumo and C.E. Yaguna, Gluino coannihilations and heavy bino dark matter, Phys. Rev. D 69 (2004) 115009 [hep-ph/0402208] [INSPIRE].

[34] D. Feldman, Z. Liu and P. Nath, Gluino NLSP, dark matter via gluino coannihilation and LHC signatures, Phys. Rev. D 80 (2009) 015007 [arXiv:0905.1148] [INSPIRE].

[35] N. Chen, D. Feldman, Z. Liu, P. Nath and G. Peim, Low mass gluino within the sparticle landscape, implications for dark matter and early discovery prospects at LHC-7, Phys. Rev. D 83 (2011) 035005 [arXiv: 1011.1246] [INSPIRE].

[36] S. Raza, Q. Shafi and C.S. Ün, NLSP gluino and NLSP stop scenarios from $b-\tau$ Yukawa unification, Phys. Rev. D 92 (2015) 055010 [arXiv:1412.7672] [inSPIRE].

[37] T.W.B. Kibble, G. Lazarides and Q. Shafi, Walls bounded by strings, Phys. Rev. D 26 (1982) 435 [INSPIRE]. 
[38] G. Lazarides and Q. Shafi, Superconducting membranes, Phys. Lett. B 159 (1985) 261 [INSPIRE].

[39] G. Lazarides and Q. Shafi, Monopoles, strings and necklaces in $\mathrm{SO}(10)$ and $E_{6}$, JHEP 10 (2019) 193 [arXiv: 1904.06880] [INSPIRE].

[40] D. Chang, R.N. Mohapatra and M.K. Parida, Decoupling parity and SU(2)-R breaking scales: a new approach to left-right symmetric models, Phys. Rev. Lett. 52 (1984) 1072 [InSPIRE].

[41] M.E. Gómez, S. Lola, R. Ruiz De Austri and Q. Shafi, Dark matter, sparticle spectroscopy and muon $(g-2)$ in $\mathrm{SU}(4)_{c} \times \mathrm{SU}(2)_{L} \times \mathrm{SU}(2)_{R}, J H E P 10$ (2018) 062 [arXiv:1806.06220] [INSPIRE].

[42] M.E. Gomez, S. Lola, R. Ruiz de Austri and Q. Shafi, Confronting SUSY GUT with dark matter, sparticle spectroscopy and muon $(g-2)$, Front. in Phys. 6 (2018) 127 [arXiv: 1806.11152] [INSPIRE].

[43] K.S. Babu and R.N. Mohapatra, Predictive neutrino spectrum in minimal $\mathrm{SO}(10)$ grand unification, Phys. Rev. Lett. 70 (1993) 2845 [hep-ph/9209215] [INSPIRE].

[44] M. Adeel Ajaib, I. Gogoladze, Q. Shafi and C.S. Un, A predictive yukawa unified $\mathrm{SO}(10)$ model: Higgs and sparticle masses, JHEP 07 (2013) 139 [arXiv:1303.6964] [INSPIRE].

[45] H. Baer, F.E. Paige, S.D. Protopopescu and X. Tata, ISAJET 7.48: a Monte Carlo event generator for $p p, \bar{p} p$, and $e^{+} e^{-}$reactions, hep-ph/0001086 [INSPIRE].

[46] J. Hisano, H. Murayama and T. Yanagida, Nucleon decay in the minimal supersymmetric SU(5) grand unification, Nucl. Phys. B 402 (1993) 46 [hep-ph/9207279] [INSPIRE].

[47] Y. Yamada, SUSY and GUT threshold effects in SUSY SU(5) models, Z. Phys. C 60 (1993) 83 [INSPIRE].

[48] J.L. Chkareuli and I.G. Gogoladze, Unification picture in minimal supersymmetric SU(5) model with string remnants, Phys. Rev. D 58 (1998) 055011 [hep-ph/9803335] [INSPIRE].

[49] D.M. Pierce, J.A. Bagger, K.T. Matchev and R.-j. Zhang, Precision corrections in the minimal supersymmetric standard model, Nucl. Phys. B 491 (1997) 3 [hep-ph/9606211] [INSPIRE].

[50] G. Bélanger, F. Boudjema, A. Pukhov and R.K. Singh, Constraining the MSSM with universal gaugino masses and implication for searches at the LHC, JHEP 11 (2009) 026 [arXiv:0906.5048] [INSPIRE].

[51] H. Baer, S. Kraml, S. Sekmen and H. Summy, Dark matter allowed scenarios for Yukawa-unified SO(10) SUSY GUTs, JHEP 03 (2008) 056 [arXiv:0801.1831] [INSPIRE].

[52] Particle Data Group collaboration, Review of particle physics, Chin. Phys. C 38 (2014) 090001 [INSPIRE].

[53] ATLAS collaboration, Observation of a new particle in the search for the Standard Model Higgs boson with the ATLAS detector at the LHC, Phys. Lett. B 716 (2012) 1 [arXiv: 1207.7214] [INSPIRE].

[54] CMS collaboration, Observation of a new boson with mass near $125 \mathrm{GeV}$ in pp collisions at $\sqrt{s}=7$ and $8 \mathrm{TeV}$, JHEP 06 (2013) 081 [arXiv:1303.4571] [INSPIRE].

[55] ATLAS, CMS collaboration, Searches for gluinos and squarks, PoS (LHCP2019) 168 [arXiv: 1909.11753] [INSPIRE]. 
[56] H. Baer and M. Brhlik, Signals for the minimal gauge-mediated supersymmetry-breaking model at the Fermilab Tevatron collider, Phys. Rev. D 55 (1997) 4463.

[57] H. Baer, M. Brhlik, D. Castano and X. Tata, $\vec{b} s \gamma$ constraints on the minimal supergravity model with large $\tan \beta$, Phys. Rev. D 58 (1998) 015007.

[58] K.S. Babu and C.F. Kolda, Higgs mediated $B^{0} \rightarrow \mu^{+} \mu^{-}$in minimal supersymmetry, Phys. Rev. Lett. 84 (2000) 228 [hep-ph/9909476] [INSPIRE].

[59] A. Dedes, H.K. Dreiner and U. Nierste, Correlation of $B_{s} \rightarrow \mu^{+} \mu^{-}$and (g-2) ( $\mu$ ) in minimal supergravity, Phys. Rev. Lett. 87 (2001) 251804 [hep-ph/0108037] [INSPIRE].

[60] J.K. Mizukoshi, X. Tata and Y. Wang, Higgs-mediated leptonic decays of $B_{s}$ and $B_{d}$ mesons as probes of supersymmetry, Phys. Rev. D 66 (2002) 115003.

[61] M.M. Roberts and S.A. Hartnoll, Pseudogap and time reversal breaking in a holographic superconductor, JHEP 08 (2008) 035 [arXiv:0805.3898] [INSPIRE].

[62] LHCb collaboration, First evidence for the decay $B_{s}^{0} \rightarrow \mu^{+} \mu^{-}$, Phys. Rev. Lett. 110 (2013) 021801 [arXiv: 1211.2674] [INSPIRE].

[63] Heavy Flavor Averaging Group collaboration, Averages of B-Hadron, C-Hadron and $\tau$-lepton properties as of early 2012, arXiv:1207.1158 [INSPIRE].

[64] Heavy Flavor Averaging Group collaboration, Averages of b-hadron, c-hadron and $\tau$-lepton properties, arXiv:1010.1589 [INSPIRE].

[65] Planck collaboration, Planck 2018 results. I. Overview and the cosmological legacy of Planck, arXiv: 1807.06205 [INSPIRE].

[66] F. Ambrogi et al., SModelS v1.1 user manual: Improving simplified model constraints with efficiency maps, Comput. Phys. Commun. 227 (2018) 72 [arXiv:1701.06586] [inSPIRE].

[67] S. Kraml et al., SModelS: a tool for interpreting simplified-model results from the LHC and its application to supersymmetry, Eur. Phys. J. C $\mathbf{7 4}$ (2014) 2868 [arXiv:1312.4175] [INSPIRE].

[68] J. Dutta, S. Kraml, A. Lessa and W. Waltenberger, SModelS extension with the CMS supersymmetry search results from Run 2, LHEP 1 (2018) 5 [arXiv: 1803. 02204] [INSPIRE].

[69] J. Heisig, S. Kraml and A. Lessa, Constraining new physics with searches for long-lived particles: Implementation into SModelS, Phys. Lett. B 788 (2019) 87 [arXiv:1808.05229] [INSPIRE].

[70] ATLAS collaboration, Interpretations of SUSY searches in ATLAS with simplified models, arXiv: 1110.0282 [INSPIRE].

[71] CMS collaboration, Interpretation of searches for supersymmetry with simplified models, Phys. Rev. D 88 (2013) 052017 [arXiv: 1301.2175] [InSPIRE].

[72] A. Djouadi, M.M. Muhlleitner and M. Spira, Decays of supersymmetric particles: the program SUSY-HIT (SUspect-SdecaY-HDECAY-InTerface), Acta Phys. Polon. B 38 (2007) 635 [hep-ph/0609292] [INSPIRE].

[73] T. Sjöstrand et al., An introduction to PYTHIA 8.2, Comput. Phys. Commun. 191 (2015) 159 [arXiv: 1410.3012] [INSPIRE].

[74] CDMS-II collaboration, Beyond the CDMS-II dark matter search: SuperCDMS, eConf C 041213 (2004) 2529 [astro-ph/0503583] [INSPIRE]. 
[75] LUX-ZEPLIN collaboration, Projected WIMP sensitivity of the LUX-ZEPLIN dark matter experiment, Phys. Rev. D 101 (2020) 052002 [arXiv: 1802.06039] [INSPIRE].

[76] Super-Kamiokande collaboration, An indirect search for WIMPs in the Sun using 3109.6 days of upward-going muons in Super-Kamiokande, Astrophys. J. 742 (2011) 78 [arXiv:1108.3384] [INSPIRE].

[77] LUX collaboration, Results on the spin-dependent scattering of weakly interacting massive particles on nucleons from the Run 3 data of the LUX experiment, Phys. Rev. Lett. 116 (2016) 161302 [arXiv: 1602.03489] [INSPIRE].

[78] CMS collaboration, Search for dark matter, extra dimensions and unparticles in monojet events in proton-proton collisions at $\sqrt{s}=8$ TeV, Eur. Phys. J. C 75 (2015) 235 [arXiv: 1408.3583] [INSPIRE].

[79] ATLAS collaboration, Search for squarks and gluinos in final states with jets and missing transverse momentum using $36 \mathrm{fb}^{-1}$ of $\sqrt{\mathrm{s}}=13$ TeV pp collision data with the ATLAS detector, Phys. Rev. D 97 (2018) 112001 [arXiv:1712.02332] [INSPIRE].

[80] ATLAS collaboration, Search for squarks and gluinos in events with an isolated lepton, jets and missing transverse momentum at $\sqrt{s}=13 \mathrm{TeV}$ with the ATLAS detector, Phys. Rev. D 96 (2017) 112010 [arXiv: 1708.08232] [INSPIRE].

[81] CMS collaboration, Search for electroweak production of charginos and neutralinos in multilepton final states in pp collision data at $\sqrt{s}=13$ TeV, CMS-PAS-SUS-16-039 (2017).

[82] CMS collaboration, Search for electroweak production of charginos and neutralinos in WH events in proton-proton collisions at $\sqrt{s}=13 \mathrm{TeV}$, JHEP 11 (2017) 029 [arXiv: 1706. 09933] [INSPIRE].

[83] CMS collaboration, Search for new physics in final states with two opposite-sign, same-flavor leptons, jets and missing transverse momentum in pp collisions at $\sqrt{s}=13 \mathrm{TeV}$, Tech. Rep. CMS-PAS-SUS-16-034, CERN, Geneva (2017).

[84] ATLAS collaboration, Search for heavy Higgs bosons decaying into two tau leptons with the ATLAS detector using pp collisions at $\sqrt{s}=13 \mathrm{TeV}$, arXiv:2002.12223 [INSPIRE].

[85] ATLAS collaboration, Search for charged Higgs bosons decaying via $H^{ \pm} \rightarrow \tau^{ \pm} \nu_{\tau}$ in the $\tau+$ jets and $\tau+$ lepton final states with $36 \mathrm{fb}^{-1}$ of pp collision data recorded at $\sqrt{s}=13 \mathrm{TeV}$ with the ATLAS experiment, JHEP 09 (2018) 139 [arXiv: 1807.07915] [INSPIRE].

[86] ATLAS collaboration, Search for heavy neutral Higgs bosons produced in association with $b$-quarks and decaying to b-quarks at $\sqrt{s}=13 \mathrm{TeV}$ with the ATLAS detector, arXiv: 1907.02749 [INSPIRE].

[87] CMS collaboration, Search for a charged Higgs boson decaying into top and bottom quarks in events with electrons or muons in proton-proton collisions at $\sqrt{\mathrm{s}}=13 \mathrm{TeV}$, JHEP 01 (2020) 096 [arXiv: 1908.09206] [INSPIRE]. 